\title{
SOME REMARKS ON A NEUMANN BOUNDARY VALUE PROBLEM ARISING IN FLUID DYNAMICS
}

\author{
PEDRO J. TORRES ${ }^{1}$
}

(Received 9 August, 2001; revised 15 July, 2002)

\begin{abstract}
It is proved that the Neumann boundary value problem, which Mays and Norbury have recently connected with a certain fluid dynamics equation, has a positive solution for any positive value of a particular parameter. Uniform bounds for the solutions and symmetry on a given range of the parameter are also introduced. The proofs include Krasnoselskii's classical fixed-point theorem on cones of a Banach space and basic comparison techniques.
\end{abstract}

\section{Introduction}

In a recent paper by Mays and Norbury [3], the Neumann boundary value problem

$$
\begin{aligned}
L u \equiv-u^{\prime \prime}+q^{2} u & =u^{2}(1+\sin x), \\
u^{\prime}(0)=0 & =u^{\prime}(\pi),
\end{aligned}
$$

was studied using analytical and numerical methods. This problem was considered as a simplified version of a fluid dynamics equation introduced by Benjamin [1]. The results in [3] are mostly of a numerical nature and show the existence of a solution if $q^{2} \in(0,10)$. It is important to obtain analytical results which could confirm and/or complement the numerical understanding of this problem [3]. This is the aim of this note. In Section 2 the existence of a solution for any value of the parameter $q>0$ is rigorously proved. The proof relies on a fixed-point theorem for completely continuous Krasnoselskii operators and the positivity of the Green's function of the linear part of the problem, as has already been observed in [3]. In Section 3 uniform bounds for the solutions are deduced as well as symmetry for a certain range of values of $q$, by using basic comparison arguments. All these results confirm the numerical evidence from [3], although the range where symmetry appears is more conservative and uniqueness remains an open problem.

\footnotetext{
'Dep. de Matemática Aplicada, Universidad de Granada, 18071 Granada, Spain; e-mail: ptorres@ugr.es.

(C) Australian Mathematical Society 2004, Serial-fee code 1446-1811/04
} 


\section{Existence of solutions}

The main result is the following.

THEOREM 2.1. Problem (1.1) has a positive solution for any positive $q$.

The proof is based on the following fixed-point theorem for cones in a Banach space [2, p. 148] and some arguments recently developed in [4].

THEOREM 2.2. Let $\mathscr{B}$ be a Banach space and let $\mathscr{P} \subset \mathscr{B}$ be a cone in $\mathscr{B}$. Assume $\Omega_{1}, \Omega_{2}$ are open subsets of $\mathscr{B}$ with $0 \in \Omega_{1}, \bar{\Omega}_{1} \subset \Omega_{2}$ and let $A: \mathscr{P} \cap\left(\Omega_{2} / \bar{\Omega}_{1}\right) \rightarrow P$ be a completely continuous operator such that one of the following conditions is satisfied:

(1) $\|A u\| \leq\|u\|, u \in \mathscr{P} \cap \partial \Omega_{1}$ and $\|A u\| \geq\|u\|, u \in \mathscr{P} \cap \partial \Omega_{2}$;

(2) $\|A u\| \geq\|u\|, u \in \mathscr{P} \cap \partial \Omega_{1}$ and $\|A u\| \leq\|u\|, u \in \mathscr{P} \cap \partial \Omega_{2}$.

Then $A$ has at least one fixed point in $\mathscr{P} \cap\left(\bar{\Omega}_{2} / \Omega_{1}\right)$.

As was observed in [3], the Green's function $k(x, y)$ of the operator $L$ with Neumann conditions is a positive and continuous function on $[0, \pi] \times[0, \pi]$. Thus problem (1.1) can be written as the fixed-point problem

$$
u(x)=\int_{0}^{\pi} k(x, y) u^{2}(y)(1+\sin y) d y \equiv A u .
$$

ProOF OF THEOREM 2.1. We follow along the lines of [4, Section 3]. If we denote

$$
m=\min k(x, y), \quad M=\max k(x, y), \quad x, y \in[0, \pi],
$$

then evidently $M>m>0$. In order to apply Theorem 2.2, let us consider the Banach space $\mathscr{B}=C([0, \pi])$ with the $L^{\infty}$-norm $\|\cdot\|_{\infty}$, and define the following cone in $\mathscr{B}$ :

$$
\mathscr{P}_{0}=\left\{u \in \mathscr{B}: \min _{x \in[0, \pi]} u(x) \geq \frac{m}{M}\|u\|_{\infty}\right\} .
$$

Let us prove that $A \mathscr{P}_{0} \subset \mathscr{P}_{0}$. For a given $u \in \mathscr{P}_{0}$, we have

$$
\begin{aligned}
\min _{x \in[0, \pi]} A u(x) & \geq \int_{0}^{\pi} m u^{2}(y)(1+\sin y) d y \\
& \geq \frac{m}{M} \int_{0}^{\pi} k(x, y) u^{2}(y)(1+\sin y) d y=\frac{m}{M} A u(x)
\end{aligned}
$$

for all $x \in[0, \pi]$, so in particular $\min _{x \in[0, \pi]} A u(x) \geq(m / M)\|A u\|_{\infty}$. 
Now let us define the open balls

$$
\Omega_{1}=\left\{u \in \mathscr{B}:\|u\|_{\infty}<\frac{1}{2 \pi M}\right\} \quad \text { and } \quad \Omega_{2}=\left\{u \in \mathscr{B}:\|u\|_{\infty}<\frac{M^{2}}{\pi m^{3}}\right\} .
$$

Clearly, $0 \in \Omega_{1}$. On the other hand, observe that the radius of $\Omega_{1}$ is less than that of $\Omega_{2}$, so $\bar{\Omega}_{1} \subset \Omega_{2}$.

Now, if $u \in \mathscr{P}_{0} \cap \partial \Omega_{1}$,

$$
\|A u\|_{\infty} \leq 2 \pi M\|u\|_{\infty}^{2}=\|u\|_{\infty}
$$

whereas if $u \in \mathscr{P}_{0} \cap \partial \Omega_{2}$,

$$
\|A u\|_{\infty} \geq m \int_{0}^{\pi} u^{2}(y)(1+\sin y) d y \geq m \int_{0}^{\pi} u^{2}(y) d y \geq \frac{m^{3}}{M^{2}} \pi\|u\|_{\infty}^{2}=\|u\|_{\infty} .
$$

Therefore (2.1), and in consequence problem (1.1), has a solution $u \in \mathscr{P}_{0} \cap\left(\bar{\Omega}_{2} / \Omega_{1}\right)$.

\section{Uniform bounds and symmetry of the solutions}

Note that from the proof of Theorem 2.1 the following bounds of the solution are deduced:

$$
\frac{m}{2 \pi M^{2}} \leq u(x) \leq \frac{M^{2}}{\pi m^{3}} .
$$

However, these bounds are valid only for this particular solution; in principle there may exist other solutions outside these limits. Our following goal is to get uniform bounds for every solution of problem (1.1).

THEOREM 3.1. There exist constants $\epsilon, C$ (only depending on $q$ ) such that any solution of problem (1.1) verifies

$$
\epsilon \leq u(x) \leq C, \quad x \in[0, \pi] .
$$

PROOF. First, it is important to consider that, as was observed in [3], every solution of (1.1) is positive. An integration of the equation gives

$$
q^{2}\|u\|_{1}=\int_{0}^{\pi} u^{2}(1+\sin x) d x \geq\|u\|_{2}^{2}
$$

and by the Cauchy-Schwartz inequality, $\|u\|_{2} \leq q^{2} \sqrt{\pi}$. Moreover,

$$
\begin{aligned}
u^{\prime}(x) & =\int_{0}^{x} u^{\prime \prime}(s) d s=\int_{0}^{x}\left(q^{2} u(s)-u^{2}(s)(1+\sin s)\right) d s<q^{2}\|u\|_{1} \leq q^{4} \pi \\
-u^{\prime}(x) & =\int_{x}^{\pi} u^{\prime \prime}(s) d s=\int_{x}^{\pi}\left(q^{2} u(s)-u^{2}(s)(1+\sin s)\right) d s<q^{2}\|u\|_{1} \leq q^{4} \pi
\end{aligned}
$$


so in consequence $\left\|u^{\prime}\right\|_{\infty}<q^{4} \pi$.

On the other hand, any non-constant solution of (1.1) must have an inflexion point, that is, there exists $\left.x_{0} \in\right] 0, \pi$ [ such that $u^{\prime \prime}\left(x_{0}\right)=0$. From this equation, it is easy to deduce that

$$
q^{2} / 2<u\left(x_{0}\right)<q^{2} .
$$

We can now deduce the upper bound $C$ as follows:

$$
u(x)=u\left(x_{0}\right)+\int_{x_{0}}^{x} u^{\prime}(s) d s<q^{2}+\pi^{2} q^{4}=: C .
$$

We still need to obtain the lower bound $\epsilon$. It will be done by comparison of $u$ with solution $\tilde{u}$ of the autonomous initial value problem

$$
\begin{gathered}
-\tilde{u}^{\prime \prime}+q^{2} \tilde{u}=\tilde{u}^{2}, \\
\tilde{u}(0)=\epsilon, \quad \tilde{u}^{\prime}(0)=0 .
\end{gathered}
$$

By continuous dependence of the solution on the initial conditions it is easy to realise that if $\epsilon$ is small enough, $\tilde{u}$ is positive, increasing, convex and $\tilde{u}<q^{4} / 4, x \in[0, \pi]$.

Evidently $\epsilon$ depends on $q$. By contradiction, let us assume that $u\left(x_{m}\right)=\min u(x)<\epsilon$. Without loss of generality, it can be assumed that $x_{m}<\pi$ (if $x_{m}=\pi$, we can continue the argument with $w(x)=u(\pi-x)$, which is also a solution of (1.1)). Let us define $z(x)=u(x)-\tilde{u}(x)$. Note that

$$
u\left(x_{m}\right)<\epsilon \leq \tilde{u}\left(x_{m}\right), \quad u^{\prime}\left(x_{m}\right)=0 \leq \tilde{u}^{\prime}\left(x_{m}\right),
$$

so $z\left(x_{m}\right)<0, z^{\prime}\left(x_{m}\right) \leq 0$. Evidently, $z$ cannot be identically zero. We are going to prove that $z(x)<0$ for all $x>x_{m}$. If this is not true, there exists $x_{1}>x_{m}$ such that $z\left(x_{1}\right)<0, z^{\prime}\left(x_{1}\right)=0$ and $z^{\prime \prime}\left(x_{1}\right) \geq 0\left(z\left(x_{1}\right)\right.$ would be a local minimum of $\left.z\right)$. Subtracting the equations,

$$
-z^{\prime \prime}\left(x_{1}\right)=z\left(x_{1}\right)\left(u\left(x_{1}\right)+\tilde{u}\left(x_{1}\right)-q^{2}\right)+\sin \left(x_{1}\right) u^{2}\left(x_{1}\right)>0,
$$

because $u\left(x_{1}\right) \leq \tilde{u}\left(x_{1}\right)<q^{2} / 4$. This is a contradiction and hence it is proved that $z(x)<0$ for all $x>x_{m}$.

As a consequence, $u(x)<q^{2} / 4$ for all $x>x_{m}$. Now, in order to finish the reasoning we only have to point out that there must be an inflexion point $u\left(x_{0}\right)$ with $x_{m}<x_{0}<\pi$, and as was observed before, $q^{2} / 2<u\left(x_{0}\right)<q^{2}$, leading to a contradiction. The consequence is that $u\left(x_{m}\right) \geq \epsilon$, and the proof is finished.

Note that constant $C$ is explicitly defined in (3.1). This information can be used to prove the symmetry of the solutions (that is, $u(x)=u(\pi-x)$ ) on a certain range of values of $q$. 
THEOREM 3.2. Let us suppose that $q$ is a positive constant such that

$$
3 q^{2}+4 \pi^{2} q^{4} \leq 1 .
$$

Then any solution of problem (1.1) is symmetric.

PROOF. Let $u_{1}$ be a solution, then it is easy to verify that $u_{2}=u_{1}(\pi-x)$ is also a solution. Our purpose is to prove that $u_{1} \equiv u_{2}$ under condition (3.2). Let us define $z=u_{1}-u_{2}$. Then $z$ is a solution of the problem

$$
\begin{gathered}
z^{\prime \prime}+\alpha(x) z=0, \\
z^{\prime}(0)=0=z^{\prime}(\pi),
\end{gathered}
$$

where $\alpha(x)=(1+\sin x)\left(u_{1}+u_{2}\right)-q^{2}$. Observe that by Theorem 3.1,

$$
u_{i}(x)<C=q^{2}+\pi^{2} q^{4}, \quad x \in[0, \pi], i=1,2 .
$$

Therefore, using condition (3.2),

$$
\alpha(x)<1, \quad x \in[0, \pi] .
$$

Let us prove that $z$ is identically zero. Let us suppose that $z$ is not the trivial solution of (3.3). Let us change to polar coordinates, $z=r \cos \theta, z^{\prime}=-r \sin \theta$. By deriving $z$ and $z^{\prime}$ we get respectively

$$
\begin{aligned}
r^{\prime} \cos \theta-r \sin (\theta) \theta^{\prime} & =-r \sin \theta, \\
-r^{\prime} \sin \theta-r \cos (\theta) \theta^{\prime} & =-\alpha(x) r \cos \theta .
\end{aligned}
$$

Multiplying the first equation by $\sin \theta$, the second one by $\cos \theta$ and adding, we obtain the equation

$$
\theta^{\prime}=\alpha(x) \cos ^{2} \theta+\sin ^{2} \theta .
$$

Now, an integration in the interval $[0, x]$ and (3.4) give

$$
\theta(x)-\theta(0)=\int_{0}^{x}\left(\alpha(s) \cos ^{2} \theta+\sin ^{2} \theta\right) d s<\int_{0}^{x}\left(\cos ^{2} \theta+\sin ^{2} \theta\right) d s=x,
$$

for all $x \in(0, \pi]$.

On the other hand, note that $z(x)=-z(\pi-x)$, and therefore $z(\pi / 2)=0$. By the Sturm comparison theorem (compare with $z^{\prime \prime}+z=0$ ), this is the unique zero of $z$ in the interval $[0, \pi]$. Besides, $z(0) z(\pi)<0$ because $z$ is not the trivial solution. We can assume without loss of generality that $z(0)>0$ (if $z(0)<0$ we work with $-z$ ). Then $\theta(0)=0$ since $z^{\prime}(0)=0$. Moreover, $z(\pi / 2)=0$ and $z^{\prime}(\pi / 2)<0$ (remember that $z$ is not the trivial solution and $z(\pi / 2)$ is the unique zero), so $\theta(\pi / 2)=\pi / 2$. But by (3.6), $\pi / 2=\theta(\pi / 2)-\theta(0)<\pi / 2$. This is a contradiction. The conclusion is that $z \equiv 0$ and therefore the proof is finished. 
A numerical computation of condition (3.2) provides $q \in 10,0.354446]$. As a final remark, the uniqueness of a positive solution on a given range of values of the parameter $q$ is strongly suggested by numerical calculations. The analytical proof remains an open problem.

\section{Acknowledgement}

I thank the referee for some useful comments that have improved the presentation of this paper. This research was supported by C.I.C.T. PB98-1294, M.E.C., Spain.

\section{References}

[1] T. B. Benjamin, "A new kind of solitary wave", J. Fluid Mech. 245 (1992) 401-411.

[2] M. A. Krasnosel'skii, Positive solutions of operator equations (Nordhoff, Groningen, 1964).

[3] L. Mays and J. Norbury, "Bifurcation of positive solutions for a Neumann boundary value problem", ANZIAM J. 42 (2001) 324-340.

[4] F. Merivenci Atici and G. Sh. Guseinov, "On the existence of positive solutions for nonlinear differential equations with periodic boundary conditions", J. Comp. Appl. Math. 132 (2001) 341356. 